\title{
SOME FIXED POINT THEOREMS IN INTUITIONISTIC FUZZY METRIC SPACES
}

\author{
SUSHIL SHARMA AND PRASHANT TILWANKAR
}

\begin{abstract}
The aim of this paper is to prove some common fixed point theorems by using the property $(S-B)$ and the notion of R-weak commutativity of type $\left(S_{p}\right)$ in intuitionistic fuzzy metric spaces. We first formulate the definition of R-weakly commuting mappings of type $\left(S_{p}\right)$ in intuitionistic fuzzy metric spaces and prove the intuitionistic fuzzy version of Pant's theorem.
\end{abstract}

\section{Introduction}

Alaca et al. [1] using the idea of intuitionistic fuzzy sets, they defined the notion of intuitionistic fuzzy metric space as Park [17] with the help of continuous t-norms and continuous t-conorms as a generalization of fuzzy metric space due to Kramosil and Michalek [11]. Further, they introduced the notion of Cauchy sequences in intuitionistic fuzzy metric spaces and proved the well known fixed point theorems of Banach [5] and Edelstein [7] extended to intuitionistic fuzzy metric spaces with the help of Grabiec [8].

Turkoglu et al. [31] introduced the concept of compatible maps and compatible maps of types $(\alpha)$ and $(\beta)$ in intuitionistic fuzzy metric spaces and gave some relations between the concepts of compatible maps and compatible maps of types $(\alpha)$ and $(\beta)$.

Gregory et al. [9], Saadati et al. [25], Singalotti et al. [24], Sharma and Deshpande [27], [28], Ciric et al. [6], Jesic [10], Kutukcu [13] and many others studied the concept of intuitionistic fuzzy metric space and its applications. Sharma and Deshpande [27] proved common fixed point theorems for finite number of mappings without continuity and compatibility on intuitionistic fuzzy metric spaces. In the study of fixed points of metric spaces Pant [18], [19], [21] has initiated work using the concept of noncompatible maps in metric spaces. The utility of study of noncompatible maps can be understood from the fact that while studying the common fixed point theorem of compatible maps we often require assumptions on the completeness of the space or the continuity of the mappings involved besides some contractive Corresponding author: Prashant Tilwankar. 2010 Mathematics Subject Classification. 47H10, 54H25.

Key words and phrases. Intuitionistic fuzzy metric space, $(S-B)$ property, R-weak commutativity of type $\left(S_{p}\right)$, Common fixed point. 
conditions but the study of noncompatible maps can be extended to the class of nonexpansive or Lipschitz type mapping pairs even without assuming continuity of the mappings involved or completeness of the space. Aamri and Moutawakil [4] introduced the property (E.A) and thus generalized the concept of noncompatible maps. The results obtained in the metric fixed point theory by using the notion of noncompatible maps or the property $(E . A)$ are very interesting.

Sharma, Kutukcu and Pathak [30] introduced the property $(S-B)$ in intuitionistic fuzzy metric spaces and proved fixed point theorems on intuitionistic fuzzy metric spaces.

In this paper we prove common fixed point theorems by introducing R-weakly commuting maps of type $\left(S_{p}\right)$ in intuitionistic fuzzy metric spaces and using the property $(S-B)$, however, without assuming either the completeness of the space or the continuity of the mappings involved. Pant [20] introduced the concept of R-weakly commuting maps in metric spaces. Later Pathak et al. [23] generalized this concept and gave the concept of R-weakly commuting maps of type $\left(A_{g}\right)$. Pant [22] defined the concept of R-weakly commuting maps of type $\left(\mathrm{A}_{g}\right)$ in the fuzzy metric space.

Here we define the concept of R-weakly commuting maps of type $\left(S_{p}\right)$ in intuitionistic fuzzy metric spaces and using the property $(S-B)$ we prove common fixed point theorems for a pair of selfmaps. Our results intuitionistically fuzzify the results of Pant [22].

\section{Preliminaries}

Definition 2.1. ([26]) A binary operation * : $[0,1] \times[0,1] \rightarrow[0,1]$ is a continuous t-norm, if * is satisfying the following conditions :

(a) * is commutative and associative;

(b) * is continuous;

(c) $a * 1=a$ for all $a \in[0,1]$;

(d) $a * b \leq c * d$ whenever $a \leq c$ and $b \leq d$, and $a, b, c, d \in[0,1]$.

Definition 2.2. ([26]) A binary operation $\diamond:[0,1] \times[0,1] \rightarrow[0,1]$ is a continuous $t$-conorm if $\diamond$ is satisfying the following conditions :

(a) $\diamond$ is commutative and associative ;

(b) $\diamond$ is continuous;

(c) $a \diamond 0=a$ for all $a \in[0,1]$;

(d) $a \diamond b \leq c \diamond d$ whenever $a \leq c$ and $b \leq d$, and $a, b, c, d \in[0,1]$.

Remark 2.1. The concept of triangular norms ( $t$-norms) and triangular conorms ( $t$-conorms) are known as the axiomatic skeletons that we use for characterizing fuzzy intersections and 
unions, respectively. These concepts were originally introduced by Menger [15] in his study of statistical metric spaces. Several examples for these concepts were proposed by many authors [12], [34].

Definition 2.3. ([1]) A 5-tuple $(X, M, N, *, \diamond)$ is said to be an intuitionistic fuzzy metric space (shortly IFM-space) if $X$ is an arbitrary set, $*$ is a continuous $t$-norm, $\diamond$ is a continuous $t$-conorm and $M, N$ are fuzzy sets on $X^{2} \times(0, \infty)$ satisfying the following conditions for all $x, y, z \in X$ and $t, s>0$,

(i) $M(x, y, t)+N(x, y, t) \leq 1$;

(ii) $M(x, y, 0)=0$;

(iii) $M(x, y, t)=1$ for all $t>0$ if and only if $x=y$;

(iv) $M(x, y, t)=M(y, x, t)$;

(v) $M(x, y, t) * M(y, z, s) \leq M(x, z, t+s)$ for all $x, y, z \in X$ and $s, t>0$;

(vi) $M(x, y, \cdot):[0, \infty) \rightarrow[0,1]$ is left continuous;

(vii) $\lim _{t \rightarrow \infty} M(x, y, t)=1$ for all $x, y i n X$;

(viii) $N(x, y, 0)=1$;

(ix) $N(x, y, t)=0$ for all $t>0$ if and only if $x=y$;

(x) $N(x, y, t)=N(y, x, t)$;

(xi) $N(x, y, t) \diamond N(y, z, s) \geq N(x, z, t+s)$ for all $x, y, z \in X$ and $s, t>0$;

(xii) $N(x, y, \cdot):[0, \infty) \rightarrow[0,1]$ is right continuous;

(xiii) $\lim _{t \rightarrow \infty} N(x, y, t)=0$ for all $x, y$ in $X$.

Then $(M, N)$ is called an intuitionistic fuzzy metric space. The functions $M(x, y, t)$ and $N(x, y, t)$ denote the degree of nearness and the degree of non-nearness between $x$ and $y$ with respect to $t$, respectively.

Remark 2.2. ([2], [3]) Every fuzzy metric space $(X, M, *)$ is an intuitionistic fuzzy metric space if $X$ of the form $(X, M, 1-M, *, \diamond)$ such that $t$-norm $*$ and $t$-conorm $\diamond$ are associated, i.e, $. x \diamond y=1-((1-x) *(1-y))$ for any $x, y \in[0,1]$. But the converse is not true.

Example 2.1. ([14]) Let $(X, d)$ be a metric space. Define $a * b=\min \{a, b\}$ and $t$-conorm $a \diamond b=$ $\max \{a, b\}$ for all $x, y \in X$ and $t>0, M_{d}(x, y, t)=\frac{t}{t+d(x, y)}$ and $N_{d}(x, y, t)=\frac{d(x, y)}{t+d(x, y)}$.

Then $(X, M, N, *, \diamond)$ is an intuitionistic fuzzy metric space. We call this intuitionistic fuzzy metric $(M, N)$ induced by the metric $\mathrm{d}$ the standard intuitionistic fuzzy metric.

Definition 2.4. ([20]) Two maps $A$ and $S$ are called $R$-weakly commuting at a point $x$ if $d(A S x, S A x) \leq$ $R d(A x, S x)$ for some $R>0$. $A$ and $S$ are called pointwise $R$-weakly commuting on $X$ if given $x$ in $X$, there exists $R>0$ such that $d(A S x, S A x) \leq R d(A x, S x)$. 
Definition 2.5. ([33]) Two mappings $A$ and $S$ of a fuzzy metric space $(X, M, *)$ into itself are $R$-weakly commuting provided there exists some real number $R$ such that $M(A S x, S A x, t) \geq$ $M(A x, S x, t / R)$ for each $x \in X$ and $t>0$.

Definition 2.6. ([32]) Let $f$ and $g$ be mappings from an intuitionistic fuzzy metric space $(X, M, N, *, \diamond)$ into itself. The mappings $f$ and $g$ are said to be $R$-weakly commuting if there exists a positive real number $R$ such that $M(f g x, g f x, t) \geq M(f x, g x, t / R), N(f g x, g f x, t) \leq$ $N(f x, g x, t / R)$ for all $x \in X$.

Definition 2.7. ([23]) Two self mappings $A$ and $S$ of a metric space $(X, d)$ are called $R$-weakly commuting of type $\left(A_{g}\right)$ if there exists some positive real number $R$ such that $d(A A x, S A x) \leq$ $R d(A x, S x)$ for all $x$ in $X$.

Definition 2.8. ([22]) Two mappings $A$ and $S$ of a fuzzy metric space $(X, M, *)$ into itself are $R$-weakly commuting of type $\left(A_{g}\right)$ provided there exists some real number $R$ such that $M(A A x, S A x, t) \geq M(A x, S x, t / R)$ for each $x \in X$ and $t>0$.

We define the following:

Definition 2.9. Two mappings $A$ and $S$ of an intuitionistic fuzzy metric space $(X, M, N, *, \diamond)$ into itself are $R$-weakly commuting of type $\left(S_{p}\right)$ provided there exists some real number $R$ such that $M(A A x, S A x, t) \geq M(A x, S x, t / R), N(A A x, S A x, t) \leq N(A x, S x, t / R)$ for each $x \in X$ and $t>0$.

Definition 2.10. ([30]) Let $S$ and $T$ be two self mappings of an intuitionistic fuzzy metric space $(X, M, N, *, \diamond)$. We say that $S$ and $T$ satisfy the property $(S-B)$ if there exists a sequence $\left\{x_{n}\right\}$ in $X$ such that $\lim _{n \rightarrow \infty} S x_{n}=\lim _{n \rightarrow \infty} T x_{n}=z$ for some $z \in X$.

Example 2.2. ([30]) Let $X=[0,+\infty)$. Define $S, T: X \rightarrow X$ by $T x=\frac{x}{5}$ and $S x=\frac{3 x}{5}$, for all $x \in X$. Consider the sequence $x_{n}=\frac{1}{n}$. Clearly $\lim _{t \rightarrow \infty} S x_{n}=\lim _{t \rightarrow \infty} T x_{n}=0$. Then $S$ and $T$ satisfy the property $(S-B)$.

Example 2.3. ([30]) Let $X=[2,+\infty)$. Define $S, T: X \rightarrow X$ by $T x=x+\frac{1}{2}$ and $S x=2 x+\frac{1}{2}$, for all $x \in X$.

Suppose the property $(S-B)$ holds; then there exists a sequence $\left\{x_{n}\right\}$ in $X$ satisfying $\lim _{n \rightarrow \infty} S x_{n}=$ $\lim _{n \rightarrow \infty} T x_{n}=z$ for some $z \in X$. Therefore $\lim _{n \rightarrow \infty} x_{n}=z-1$ and $\lim _{n \rightarrow \infty} x_{n}=\frac{z-1}{2}$. Then $z=\frac{1}{2}$, which is a contradiction since $\frac{1}{2} \notin X$. Hence $S$ and $T$ do not satisfy the property $(S-B)$.

Remark 2.3. It is clear from the definition of Mishra et al. [16] and Sharma and Deshpande [29] that two self mappings $S$ and $T$ of an intuitionistic fuzzy metric space $(X, M, N, *, \diamond)$ will be noncompatible if there exists at least one sequence $\left\{x_{n}\right\}$ in $X$ such that $\lim _{n \rightarrow \infty} S x_{n}=$ 
$\lim _{n \rightarrow \infty} T x_{n}=z$ for some $z \in X$, but $\lim _{n \rightarrow \infty} M\left(S T x_{n}, T S x_{n}, t\right)$ is either not equal to 1 or nonexistent and $\lim _{n \rightarrow \infty} N\left(S T x_{n}, T S x_{n}, t\right)$ is either not equal to 0 or non-existent. Therefore two noncompatible self mappings of an intuitionistic fuzzy metric space $(X, M, N, *, \diamond)$ satisfy the property $(S-B)$.

Now we prove the intuitionistic fuzzy version of Pant's theorem [22] by using the property $(S-B)$ and the notion of R-weak commutativity of type $\left(S_{p}\right)$ in intuitionistic fuzzy metric spaces, however, without assuming either the completeness of the space or the continuity of the mappings involved.

\section{Main Results}

Theorem 3.1. Let $(X, M, N, *, \diamond)$ be an intuitionistic fuzzy metric space and $f$ and $g$ be pointwise $R$-weakly commuting self maps of type $\left(S_{p}\right)$ of $X$ satisfying the following conditions:

(i) $f(X) \subset g(X)$,

(ii) $M(f x, f y, t) \geq \min \{M(g x, g y, t h), M(f x, g x, t h), M(f y, g y, t h), M(f y, g x, t h), M(f x, g y, t h)\}$ and $N(f x, f y, t) \leq \max \{N(g x, g y, t h), N(f x, g x, t h), N(f y, g y, t h), N(f y, g x, t h), N(f x, g y, t h)\}$, $0 \leq h<1, t>0$.

If $f$ and $g$ satisfy the property $(S-B)$ and the range of either of $f(X)$ or $g(X)$ is a complete subspace of $X$, then $f$ and $g$ have a unique common fixed point.

Proof. Since $f$ and $g$ satisfy the property $(S-B)$, there exists a sequence $\left\{x_{n}\right\}$ in $X$ such that (1) $\lim _{n \rightarrow \infty} f x_{n}=\lim _{n \rightarrow \infty} g x_{n}=z$ for some $z$ in $X$. Since $z \in f(X)$ and $f(X) \subset g(X)$, there exists some point $u$ in $X$ such that $z=g u$ where $z=\lim _{n \rightarrow \infty} g x_{n}$. If $f u \neq g u$, the inequality

$$
\begin{gathered}
M\left(f x_{n}, f u, t\right) \geq \min \left\{M\left(g x_{n}, g u, t h\right), M\left(f x_{n}, g x_{n}, t h\right), M(f u, g u, t h), M\left(f u, g x_{n}, t h\right),\right. \\
\left.M\left(f x_{n}, g u, t h\right)\right\},
\end{gathered}
$$

and

$$
\begin{gathered}
N\left(f x_{n}, f u, t\right) \leq \max \left\{N\left(g x_{n}, g u, t h\right), N\left(f x_{n}, g x_{n}, t h\right), N(f u, g u, t h), N\left(f u, g x_{n}, t h\right),\right. \\
\left.N\left(f x_{n}, g u, t h\right)\right\},
\end{gathered}
$$

Letting $n \rightarrow \infty$, we have

$$
\begin{aligned}
M(g u, f u, t) & \geq \min \{M(g u, g u, t h), M(g u, g u, t h), M(f u, g u, t h), M(f u, g u, t h), \\
& M(g u, g u, t h)\}, \\
& =M(g u, f u, t h)
\end{aligned}
$$

and 


$$
\begin{aligned}
N(g u, f u, t) & \leq \max \{N(g u, g u, t h), N(g u, g u, t h), N(f u, g u, t h), N(f u, g u, t h), \\
& N(g u, g u, t h)\}, \\
& =N(g u, f u, t h)
\end{aligned}
$$

Hence $f u=g u$.

Since $f$ and $g$ are $R$-weak commutating of type $\left(S_{p}\right)$, there exists $R>0$ such that

$$
M(f f u, g f u, t) \geq M(f u, g u, t / R)=1
$$

and

$$
N(f f u, g f u, t) \leq N(f u, g u, t / R)=0,
$$

that is, $f f u=g f u$ and $f f u=f g u=g f u=g g u$.

If $f u \neq f f u$, using (ii), we have

$$
\begin{aligned}
M(f u, f f u, t) & \geq \min \{M(g u, g f u, t h), M(f u, g u, t h), M(f f u, g f u, t h), M(f f u, g f u, t h), \\
& =M(g u, f f u, t h)\}, \\
& M(f u, f f u, t h)
\end{aligned}
$$

and

$$
\begin{aligned}
N(f u, f f u, t) & \leq \max \{N(g u, g f u, t h), N(f u, g u, t h), N(f f u, g f u, t h), N(f f u, g f u, t h), \\
& =N(f u, f f u, t h)
\end{aligned}
$$

a contradiction. Hence $f u=f f u$ and $f u=f f u=f g u=g f u=g g u$.

Hence $f u$ is a common fixed point of $f$ and $g$. The case when $f(X)$ is a complete subspace of $X$ is similar to the above case since $f(X) \subset g(X)$. Hence we have the theorem.

We now give an example to illustrate the above theorem.

Example 3.4. Let $X=[2,20]$. Define $f, g: X \rightarrow X$ as

$$
\begin{array}{lll}
f x=2 & \text { if } x=2 \text { or }>5, \quad f x=6 & \text { if } 2<x \leq 5 \\
g 2=2, & g x=x+4 & \text { if } 2<x \leq 5, \\
g x=(4 x+10) / 15 & \text { if } x>5
\end{array}
$$

also we define

$$
M(f x, g y, t)=\frac{t}{t+d(f x, g y)} \text { and } \quad N(f x, g y, t)=\frac{d(f x, g y)}{t+d(f x, g y)}
$$

for every $x, y \in X$ and $t>0$.

Then $f$ and $g$ satisfy all the conditions of the above theorem and have a common fixed point at $x=2$. In this example $f(X)=\{2\} \cup\{6\}$ and $g(X)=[2,9]$. It may be seen that $f(X) \subset$ $g(X)$. It can be verified also that $f$ and $g$ are pointwise $R$-weakly commuting maps of type $\left(S_{p}\right)$ and satisfy the property $(S-B)$. 
Theorem 3.2. Let $(X, M, N, *, \diamond)$ be an intuitionistic fuzzy metric space and $f$, $g$ be noncompatible pointwise $R$-weakly commuting self maps of type $(S p)$ of $X$ satisfying the following conditions:

(i) $f(X) \subset g(X)$,

(ii) $M(f x, f y, t) \geq \min \{M(g x, g y, t h), M(f x, g x, t h), M(f y, g y, t h), M(f y, g x, t h), M(f x, g y, t h)\}$, and $N(f x, f y, t) \leq \max \{N(g x, g y, t h), N(f x, g x, t h), N(f y, g y, t h), N(f y, g x, t h), N(f x, g y, t h)\}$, $0 \leq h<1, t>0$.

If the range of $f$ or $g$ is a complete subspace of $X$, then $f$ and $g$ have a unique common fixed point and the fixed point is the point of discontinuity.

Proof. Since $f$ and $g$ are non-compatible maps, there exists a sequence $\left\{x_{n}\right\}$ in $X$ such that (1) $\lim _{n \rightarrow \infty} f x_{n}=\lim _{n \rightarrow \infty} g x_{n}=z$ for some $z$ in $X$, either $\lim _{n \rightarrow \infty} M\left(f g x_{n}, g f x_{n}, t\right) \neq 1$ and $\lim _{n \rightarrow \infty} N\left(f g x_{n}, g f x_{n}, t\right) \neq 0$ or the limit does not exist. Since $z \in f(X)$ and $f(X) \subset g(X)$, there exists some point $u$ in $X$ such that $z=g u$ where $z=\lim _{n \rightarrow \infty} g x_{n}$. If $f u \neq g u$, the inequality

$$
\begin{gathered}
M\left(f x_{n}, f u, t\right) \geq \min \left\{M\left(g x_{n}, g u, t h\right), M\left(f x_{n}, g x_{n}, t h\right), M(f u, g u, t h), M\left(f u, g x_{n}, t h\right),\right. \\
\left.M\left(f x_{n}, g u, t h\right)\right\},
\end{gathered}
$$

and

$$
\begin{gathered}
N\left(f x_{n}, f u, t\right) \leq \max \left\{N\left(g x_{n}, g u, t h\right), N\left(f x_{n}, g x_{n}, t h\right), N(f u, g u, t h), N\left(f u, g x_{n}, t h\right),\right. \\
\left.N\left(f x_{n}, g u, t h\right)\right\} .
\end{gathered}
$$

Letting $n \rightarrow \infty$, we have

$$
M(g u, f u, t) \geq M(g u, f u, t h)
$$

and

$$
N(g u, f u, t) \leq N(g u, f u, t h) .
$$

Hence $f u=g u$.

Since $f$ and $g$ are $R$-weak commutating of type $\left(S_{p}\right)$, there exists $R>0$ such that

$$
M(f f u, g f u, t) \geq M(f u, g u, t / R)=1
$$

and

$$
N(f f u, g f u, t) \leq N(f u, g u, t / R)=0,
$$

that is, $f u=g f u$ and $f f u=f g u=g f u=g g u$. If $f u \neq f f u$, using (ii), we have

$$
M(f u, f f u, t) \geq \min \{M(g u, g f u, t h), M(f u, g u, t h), M(f f u, g f u, t h), M(f f u, g f u, t h),
$$




$$
\begin{aligned}
& M(g u, f f u, t h)\}, \\
& =M(f u, f f u, t h)
\end{aligned}
$$

and

$N(f u, f f u, t) \leq \max \{N(g u, g f u, t h), N(f u, g u, t h), N(f f u, g f u, t h), N(f f u, g f u, t h)$,

$$
\begin{aligned}
& N(g u, f f u, t h)\}, \\
= & N(f u, f f u, t h)
\end{aligned}
$$

a contradiction. Hence $f u=f f u$ and $f u=f f u=f g u=g f u=g g u$.

Hence $f u$ is a common fixed point of $f$ and $g$. The case when $f(X)$ is a complete subspace of $\mathrm{X}$ is similar to the above case since $f(X) \subset g(X)$.

Now we show that $f$ and $g$ are discontinuous at the common fixed point $z=f u=g u$. If possible, suppose $f$ is continuous. Then considering the sequence $\left\{x_{n}\right\}$ of (1) we get $\lim _{n \rightarrow \infty} f f x_{n}=$ $f z=z$. $R$-weak commutativity of type $\left(S_{p}\right)$ implies that

$$
M\left(f f x_{n}, g f x_{n}, t\right) \geq M\left(f x_{n}, g x_{n}, t / R\right)=1
$$

and

$$
N\left(f f x_{n}, g f x_{n}, t\right) \leq N\left(f x_{n}, g x_{n}, t / R\right)=0,
$$

which on letting $n \rightarrow \infty$ yields $\lim _{n \rightarrow \infty} g f x_{n}=f z=z$.

This, in turn, yields $\lim _{n \rightarrow \infty} M\left(f g x_{n}, g f x_{n}, t\right)=1$ and $\lim _{n \rightarrow \infty} N\left(f g x_{n}, g f x_{n}, t\right)=0$.

This contradicts the fact that $\lim _{n \rightarrow \infty} M\left(f g x_{n}, g f x_{n}, t\right)$ and $\lim _{n \rightarrow \infty} N\left(f g x_{n}, g f x_{n}, t\right)$ is either nonzero or nonexistent for the sequence $\left\{x_{n}\right\}$ of (1). Hence $f$ is discontinuous at the fixed point. Next, suppose that $g$ is continuous, then for the sequence $\left\{x_{n}\right\}$ of (1), we get $\lim _{n \rightarrow \infty} g f x_{n}=g z=z$ and $\lim _{n \rightarrow \infty} g g x_{n}=g z=z$. In view of these limits, the inequality

$$
\begin{aligned}
M\left(f x_{n}, f g x_{n}, t\right) \geq & \min \left\{M\left(g x_{n}, g g x_{n}, t h\right), M\left(f x_{n}, g g x_{n}, t h\right), M\left(f g x_{n}, g g x_{n}, t h\right),\right. \\
& \left.M\left(f g x_{n}, g x_{n}, t h\right), M\left(f x_{n}, g g x_{n}, t h\right)\right\}, \\
& =M\left(f x_{n}, f g x_{n}, t h\right)
\end{aligned}
$$

and

$$
\begin{aligned}
N\left(f x_{n}, f g x_{n}, t\right) \leq & \max \left\{N\left(g x_{n}, g g x_{n}, t h\right), N\left(f x_{n}, g g x_{n}, t h\right), N\left(f g x_{n}, g g x_{n}, t h\right),\right. \\
& \left.N\left(f g x_{n}, g x_{n}, t h\right), N\left(f x_{n}, g g x_{n}, t h\right)\right\}, \\
& =N\left(f x_{n}, f g x_{n}, t h\right)
\end{aligned}
$$

yield a contradiction unless $\lim _{n \rightarrow \infty} f g x_{n}=f z=g z$. But $\lim _{n \rightarrow \infty} f g x_{n}=g z$ and $\lim _{n \rightarrow \infty} g f x_{n}=$ $g z$ contradicts the fact that $\lim _{n \rightarrow \infty} M\left(f g x_{n}, g f x_{n}, t\right)$ and $\lim _{n \rightarrow \infty} N\left(f g x_{n}, g f x_{n}, t\right)$ is either nonzero or nonexistent. Thus both $f$ and $g$ are discontinuous at their common fixed point. Hence we have the theorem.

We now give an example to illustrate the above theorem. 
Example 3.5. Let $X=[2,20]$. Define $f, g: X \rightarrow X$ as

$$
\begin{array}{lrr}
f x=2 & \text { if } x=2 \text { or }>5, \quad f x=6 & \text { if } 2<x \leq 5 \\
g 2=2, \quad g x=7 & \text { if } 2<x \leq 5, \\
g x=(4 x+10) / 15 & \text { if } x>5
\end{array}
$$

also we define

$$
M(f x, g y, t)=\frac{t}{t+d(f x, g y)} \text { and } \quad N(f x, g y, t)=\frac{d(f x, g y)}{t+d(f x, g y)}
$$

for every $x, y \in X$ and $t>0$.

Then $f$ and $g$ satisfy all the conditions of the above theorem and have a common fixed point at $x=2$.

In this example $f(X)=\{2\} \cup\{6\}$ and $g(X)=[2,6] \cup\{7\}$. It may be seen that $f(X) \subset g(X)$.

It can be verified also that $f$ and $g$ are pointwise $R$-weakly commuting maps of type $\left(S_{p}\right)$. To see that $f$ and $g$ are noncompatible, let us consider a sequence $\left\{x_{n}=5+1 / n: n>1\right\}$, then $\lim _{n \rightarrow \infty} f x_{n}=2, \lim _{n \rightarrow \infty} g x_{n}=2, \lim _{n \rightarrow \infty} f g x_{n}=6$ and $\lim _{n \rightarrow \infty} g f x_{n}=2$. Hence $f$ and $g$ are noncompatible.

\section{References}

[1] C. Alaca, D. Turkoglu and C. Yildiz, Fixed points in intuitionistic fuzzy metric spaces, Choas, Solitons \& Fractals, 29 (2006), 1073-1078.

[2] C. Alaca, I. Altun and D. Turkoglu, On compatible mappings of type (I) and (II) in intuitionistic fuzzy metric spaces, Commun. Korean Math. Soc., 23(2008), 427-446.

[3] C. Alaca, D. Turkoglu and C. Yildiz, Common fixed points of compatible maps in intuitionistic fuzzy metric spaces, Southeast Asian Bull. Math., 32(2008), 21-33.

[4] M. Aamri and D. EI Moutawakil, Some new common fixed point theorems under strict contractive conditions, J.Math. Anal. Appl., 270(2002), 181-188.

[5] S. Banach, Theoriles operations Linearies Manograie Mathematyezne, Warsaw, Poland, 1932.

[6] L. Ciric, S. Jesic and J. Ume, The existence theorems for fixed and periodic points of nonexpansive mappings in intuitionistic fuzzy metric spaces, Choas, Solitons \& Fractals, 37(2008), 781-791.

[7] M. Edelstein, On fixed and periodic points under contractive mappings, J. London Math. Soc., 37(1962), 7479.

[8] M. Grabiec, Fixed points in fuzzy metric spaces, Fuzzy Sets and Systems, 27(1988), 385-389.

[9] V. Gregory, S. Romaguera and P. Veeramani, A note on intuitionistic fuzzy metric spaces, Chaos, Solitons \& Fractals, 28 (2006), 902-905.

[10] S. Jesic, Convex structure, normal structure and a fixed point theorem in intuitionistic fuzzy metric spaces, Chaos, Solitons \& Fractals, 41(2009), 292-301.

[11] O. Karmosil and J. Michalek, Fuzzy metric and statistical metric spaces, Kybernetica, 11(1975), 326-334.

[12] E. P. Klement, R. Mesiar and E. Pap, Triangular Norms, Kluwer Academic Pub. Trends in Logic 8, Dordrecht 2000 . 
[13] S. Kutukcu, Weak Compatibility and common coincidence points in intuitionistic fuzzy metric spaces, Southeast Asian Bulletin of Mathematics, 32(2008), 1081-1089.

[14] S. Kutukcu, C. Yildiz and D. Turkoglu, Fixed points of contractive mappings in intuitionistic fuzzy metric spaces, J. Comput. Anal. Appl., 9(2007), 181-193.

[15] K. Menger, Statistical metrices, Proc. Nat. Acad. Sci., 28(1942), 535-537.

[16] S. N. Mishra, N. Sharma and S. L. Singh, Common fixed points of maps on fuzzy metric spaces, Internat. J. Math. Math. Sci., 17(1994), 253-258.

[17] J. H. Park, Intuitionistic fuzzy metric spaces, Choas, Solitons \& Fractals, 22(2004), 1039-1046.

[18] R. P. Pant, Common fixed points of contractive maps, J. Math. Anal. Appl., 226(1998), 251-258.

[19] R. P. Pant, Noncompatible mappings and common fixed points, Soochow J. Math., 26(2000), 29-35.

[20] R. P. Pant, Common fixed points of noncommuting mappings, J. Math. Anal. Appl., 188(1994), 436-440.

[21] R. P. Pant, Common fixed points of Lipschitz type mapping Pairs, J. Math. Anal. Appl., 240(1999), $280-283$.

[22] V. Pant, Some fixed point theorems in fuzzy metric space, Tamkang J. Math., 40(2009), 59-66.

[23] H. K. Pathak, Y. J. Cho and S. M. Kang, Remarks on R-weakly commuting mappings and common fixed point theorems, Bull. Korean Math. Soc., 34(1997), 247-257.

[24] LDJ Sigalotti and A. Mejias, On El Naschie's conjugate complex time, fractal E( $\infty)$ space-time and faster-thanlight particles, Int. J. Nonlinear Sci. Number Simul., 7, (2006), 467-472.

[25] R. Saadati, S. Sedghi and N. Shobe, Modified intuitionistic fuzzy metric spaces and some fixed point theorems, Chaos, Solitons \& Fractals, 38(2008), 36-47.

[26] B. Schweizer and A. Sklar, Statistical metric spaces, Pacific Journal Math., 10(1960), 314-334.

[27] Sushil Sharma and B. Deshpande, Common fixed point theorems for finite number of mappings without continuity and compatibility on intuitionistic fuzzy metric spaces, Choas, Solitons \& Fractals, 40(2009), 22422256.

[28] Sushil Sharma and B. Deshpande, Compatible mappings of type (I) and (II) on intuitionistic in consideration of common fixed point, Comm. Korean Math. Soc., 24(2009), 197-214.

[29] Sushil Sharma and B. Deshpande, Discontinuity and weak compatibility in fixed point consideration on noncomplete fuzzy metric spaces, J. Fuzzy Math., 11(2003), 671-686.

[30] Sushil Sharma, S. Kutukcu and A. Pathak, Common fixed point theorems for weakly compatible mappings in intuitionistic fuzzy metric spaces, J. Fuzzy Math., 17(2009), 225-240.

[31] D. Turkoglu, C. Alaca and C. Yildiz, Compatible maps and compatible maps of types $(\alpha)$ and $(\beta)$ in intuitionistic fuzzy metric spaces, Demonstratio Math., 39 (2006), 671-684.

[32] D. Turkoglu, C. Alaca, Y. J. Cho and C. Yildiz, Common fixed point theorems in intuitionistic fuzzy metric spaces, J. Appl. Math. and Computing, 22 (2006), 411-424.

[33] R. Vasuki, Common fixed point for R-weakly commuting maps in fuzzy metric spaces, Indian J. Pure Appl. Math., 30(1999), 419-423.

[34] R. R. Yager, On a class of weak triangular norm operators, Information Sciences, 96(1997), 47-78.

${ }^{1}$ Department of Mathematics, Madhav Science College, Vikram University, Ujjain-456010, India.

E-mail: sksharma2005@yahoo.com

${ }^{2}$ Department of Mathematics, Shri Vaishnav Institute of Management, Indore-452009, India.

E-mail: prashant_tilwankar@yahoo.com 